\title{
Crisis addisoniana como primera manifestación de insuficiencia suprarrenal en paciente diagnosticado de cáncer de pulmón
}

\author{
A. MUÑOZ, J. OÑATE*, J. M. MAÑÉ, I. RUBIO, R. FERNÁNDEZ, J. R. BARCELÓ, \\ G. LÓPEZ VIVANCO
}

Servicio de Oncología Médica y *Servicio de Urgencias del Hospital de Cruces. Osakidetza/SVS. Barakaldo. Bizkaia.

\author{
ADDISONIAN CRISIS AS INITIAL MANIFESTATION OF ADRENAL \\ INSUFFICIENCY IN A PATIENT WITH LUNG CANCER
}

\begin{abstract}
RESUMEN
La insuficiencia suprarrenal primaria o enfermedad de Addison es, actualmente, una patología infrecuente que se ha descrito asociada a múltiples causas.

Presentamos el caso de un paciente de 50 años diagnosticado de adenocarcinoma de pulmón con metástasis suprarrenales bilaterales en tratamiento quimioterápico con mitomicina, ifosfamida y cisplatino (MIC), que se diagnostica de insuficiencia suprarrenal a raíz de cuadro clínico compatible con crisis addisoniana florida.

Las manifestaciones clínicas de insuficiencia suprarrenal pueden pasar inadvertidas en muchas ocasiones, por ser inespecíficas y fácilmente enmascaradas por otras causas. Se ha descrito asociada a muy diversos tumores, sobre todo a linfomas no Hodgkin. Parece existir una discordancia entre el número de pacientes con destrucción metastásica adrenal bilateral y el número de casos de insuficiencia clínica descritos, lo que puede deberse a diversas causas. Una vez sospechada la confirmación diagnóstica y el tratamiento sustitutivo son relativamente sencillos. Se hace una revisión de las distintas etiologías de la enfermedad de Addison, haciendo especial hincapié en las relacionadas con enfermos neoplásicos.
\end{abstract}

PALABRAS CLAVE: Enfermedad de Addison. Adenocarcinoma de pulmón. Metástasis suprarrenales.

\begin{abstract}
Adrenal insufficiency or Addison's disease is actually a rare illness associated with numerous pathologies. We describe the case of a fifty years old male with lung adenocarcinoma and metastasis in both adre nal glands, who was receiving chemotherapy with mytomicin, ifosfamide and cisplatin (MIC), and was diagnosed of adrenal insufficiency as a result of acute episode addisonian crisis.

Many times, the clinic symptoms of adrenal insufficiency can go unnoticed due to its low specifity and to mixing up with other syndromes. Hypoadrenalism has been described in association with many tumours, specially with non-Hodgkin's lymphoma. It seems that there is a discor dance between the number of patients with bilateral metastatic adrenal destruction and the documentated cases of clinic insufficiency. Once the adrenal failure is suspected, the diagnosis and hormone replacement tre atment are really easy. Addison's disease ethiologies are revised putting special emphasis on those related with cancer patients.
\end{abstract}

KEY WORDS: Addison's disease. Lung adenocarcinoma. Metastasis adrenal glands.

Muñoz A, Oñate J, Mañé JM, Rubio I, Fernández R, Barceló JR, López Vivanco G. Crisis Addisoniana como primera manifestación de insuficiencia suprarrenal en paciente diagnosticado de cáncer de pulmón. An Med Interna (Madrid) 2001; 18: 35-37.

\section{INTRODUCCIÓN}

La insuficiencia corticosuprarrenal primaria o enfermedad de Addison es una entidad rara que afecta a menos de 60 personas por millón de habitantes $(1,2)$, al contrario que el déficit secundario por inhibición de la producción de hormona corticotropa hipofisaria (ACTH) $(1,3)$, debido casi siempre a la supresión del tratamiento crónico con esteroides exógenos.

Antiguamente, la destrucción de las glándulas suprarrenales por tuberculosis (TBC) y otras enfermedades granulomatosas crónicas suponían la principal causa de déficit corticosuprarrenal primario. En la actualidad, la causa más frecuente $(75 \%)$ es la atrofia idiopática por fenómenos autoinmunes sobre la corteza suprarrenal, de forma aislada, o en el contexto de los síndromes de deficiencia poliendocrina tipo I y II, pasando la etiología TBC a un segundo plano (20\%). Rara vez la enfermedad de Addison se debe a infecciones oportunistas, sobre todo asociadas a SIDA, hemorragias bilaterales, ablación quirúrgica, amiloidosis, sarcoidosis, hemocromatosis, adrenoleucodistrofia, adrenomieloneuropatía, radioterapia abdominal, fármacos inhibidores enzimáticos, siendo los más freceuntes el ketoconazol, la aminoglutetimida y el mitotano, fármacos aceleradores del catabolismo esteroideo, como la rifampicina, la fenitoína o el fenobarbital, e infiltración metastásica bilateral $(2,4)$.

Trabajo aceptado: 24 de Mayo de 1999

Correspondencia: A. Muñoz Llarena. Servicio de Oncología Médica. Hospital de Cruces. Osakidetza/SVS. Plaza de Cruces, s/n. Barakaldo 48903. Bizkaia. 


\section{CASO APORTADO}

Presentamos el caso de un varón de 50 años, jardinero de exteriores y fumador de 20 paquetes/año, diagnosticado de un adenocarcinoma de pulmón por punción transparietal de nódulo pulmonar solitario de $3 \mathrm{~cm}$, con afectación metastásica suprarrenal bilateral, confirmada histológicamente por punción de ambas glándulas, que tenían un tamaño de $4 \mathrm{~cm}$ (Fig. 1). Por ello, había iniciado tratamiento con poliquimioterapia según esquema MIC (Mitomicina-C 6 $\mathrm{mg} / \mathrm{m}^{2}$ + Ifosfamida $3 \mathrm{~g} / \mathrm{m}^{2}$ + cisplatino $50 \mathrm{mg} / \mathrm{m}^{2}$ ) cada 21 días, con ondansetrón $8 \mathrm{mg}$ y dexametasona $8 \mathrm{mg}$ el día 1 como pauta antiemética, de los que había recibido 3 ciclos estando pendiente de TAC toraco-abdominal para evaluación de respuesta. Tanto en el momento de iniciar el tratamiento quimioterápico como en los controles previos a la administración de cada ciclo el paciente presentaba una analítica básica normal.

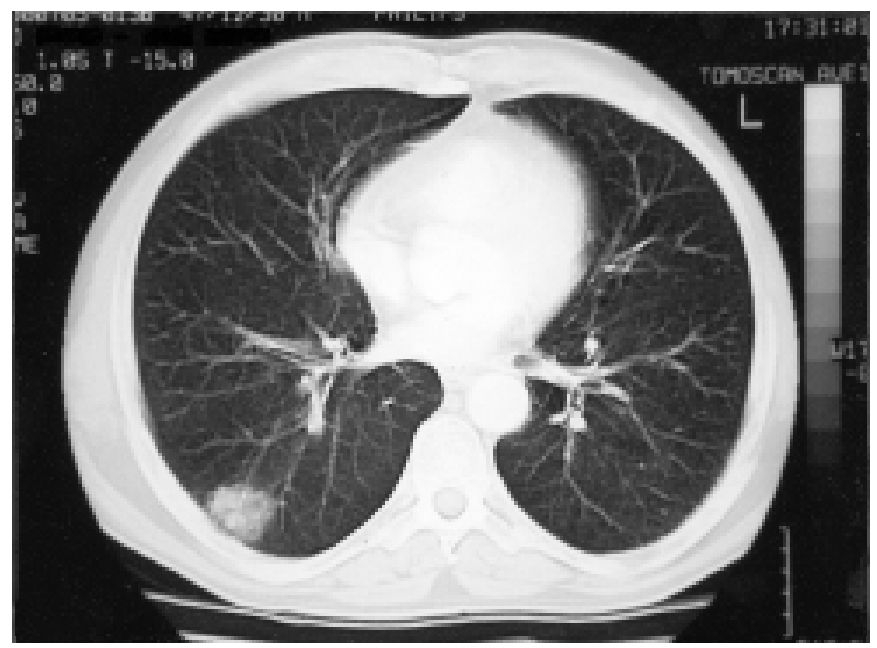

Fig. 1. Adenocarcinoma pulmón en LSD.

Acude al Servicio de Urgencias de nuestro hospital por cuadro de 10 días de evolución de diarrea acuosa de 3-4 deposiciones/día, vómitos persistentes, anorexia y astenia importante sin fiebre ni dolor abdominal, que había comenzado dos días después de administración del tercer ciclo de quimioterapia. El paciente mantenía un aceptable nivel de conciencia a pesar de presentar una tensión arterial de $70 / 50 \mathrm{~mm}$, con una frecuencia cardiaca de 108 pulsaciones por minuto y una temperatura axilar de $36,5{ }^{\circ} \mathrm{C}$. A la exploración física destacaba hiperpigmentación cutánea que el paciente achacaba a su trabajo en exteriores. Los resultados de laboratorio eran: sodio $130 \mathrm{mEq} / \mathrm{L}$, potasio $5,51 \mathrm{mEq} / \mathrm{L}$, Urea $116 \mathrm{mg} / \mathrm{dL}$, Osmolaridad $297,5 \mathrm{mOsm} / \mathrm{kg}$, cloro $97,9 \mathrm{mg} / \mathrm{dL}$, glucosa $107 \mathrm{mg} / \mathrm{dL}$, creatinina $2,50 \mathrm{mg} / \mathrm{dL}$, calcio 10,8 mg/dL, pH 7,35, $\mathrm{PaCO}_{2} 31,4 \mathrm{mmHg}, \mathrm{PaO}_{2}$ $98 \mathrm{mmHg}$, Bicarbonato $17,4 \mathrm{mmol} / \mathrm{L}$ con $\mathrm{EB}-6,40 \mathrm{mmoL} / \mathrm{L}$. Se inició tratamiento mediante reposición volumétrica con hidratación y expansores sin elevación de la tensión arterial, por lo que ante la sospecha clínica de insuficiencia suprarrenal se comenzó tratamiento con $100 \mathrm{mg}$ de hidrocortisona intravenosa, normalizándose las cifras hasta $100 / 70 \mathrm{mmHg}$ a las tres horas. Con tratamiento esteroideo y de soporte la sintomatología cedió en 24 horas, normalizando función renal y parámetros hidroelectrolíticos en dos días.

Los niveles de cortisol basal a las 8:00 a.m., extraídos previamente al inicio del tratamiento corticoideo, fueron de 2,3 microgr/dL (N: 6-24 microgr/dL). No se realizaron determinación de ACTH basal ni pruebas dinámicas. En TAC toracoabdominal se evidenció estabilización de enfermedad a nivel pulmonar con progresión suprarrenal bilateral, que presentaban un tamaño de $6 \mathrm{~cm}$ con áreas de necrosis (Fig. 2).

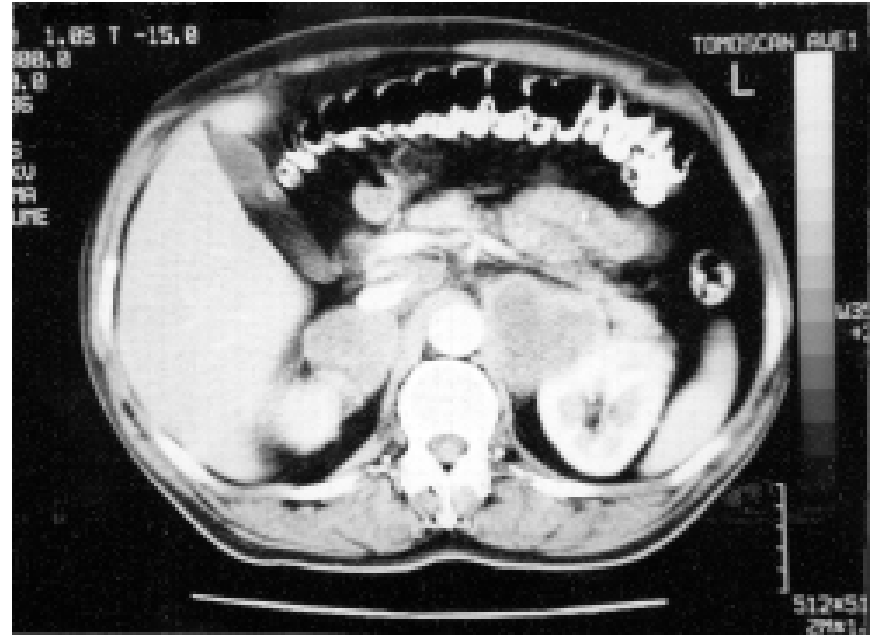

Fig. 2. M etástasis suprarrenales bilaterales.

\section{DISCUSION}

Clínicamente, la insuficiencia suprarrenal cursa con astenia, pérdida ponderal, hiperpigmentación, náuseas y vómitos, hipotensión, dolor abdominal, apetencia por alimentos salados, diarrea y síncopes $(2,3)$. Los datos de laboratorio más llamativos son hiponatremia, hiperpotasemia, hipercalcemia e hipoglucemia $(2,4)$.

Estos síntomas y hallazgos analíticos pueden pasar fácilmente inadvertidos en el contexto de pacientes con cáncer metastásico, sobre todo si están recibiendo tratamiento citostático (5). Habitualmente, la sintomatología se instaura de forma progresiva, pero en ocasiones pueden debutar como una crisis addisoniana florida, casi siempre en pacientes con fallo glandular crónico sometido a un agente estresante $(1,6)$. El diagnóstico de insuficiencia suprarrenal debe hacerse mediante determinaciones basales de cortisol y ACTH y, en caso de dudas, con el test de estimulación con cosintropina intravenosa. Niveles basales de cortisol (entre las 8:00 y 9:00 a.m.) menores de 3 microgramos por decilitro son diagnósticos de insuficiencia suprarrenal, pudiendo obviarse la realización de pruebas dinámicas de estimulación $(1,7)$. Una vez demostrada la hipofunción suprarrenal debe intentar filiarse etiológicamente, para lo cual resulta muy útil la TAC abdominal $(8,9)$, y en casos seleccionados la RM, seguidos de punción transparietal si fuera necesario.

La afectación metastásica de las glándulas suprarrenales es relativamente frecuente, y no es raro que se encuentren afectadas de modo bilateral. Aún así, revisando la literatura, se constata que la aparición de insuficiencia suprarrenal por invasión metastásica bilateral es poco frecuente, siendo la mayor serie que hemos encontrado de 13 pacientes (10). Se han descrito casos asociados a linfomas no Hodgkin $(11,12)$ y Hodgkin (13), carcinoma broncógeno (14), seminoma (15), carcinoma transicional de vejiga (16), cáncer pancreático (17), colo-rectal (18), gástrico $(19,20)$, esofágico (20) y renal (21). La descripción de crisis addisoniana como debut de fallo suprarrenal por esta etiología es prácticamente anecdótica $(6,12,22,23)$.

Probablemente, el reducido número de casos de enfermedad de Addison en relación al número de enfermos con ambas 
suprarrenales afectas se deba a tres causas: primero, la baja sospecha diagnóstica por solapamiento de síntomas con los propios del cáncer o los secundarios a toxicidad de tratamiento (5); segundo, por la necesidad de que estén destruidas más del $90 \%$ del parénquima glandular para que la hipofunción sea clínicamente evidente (6), tercero, por el extendido uso de los corticoides como tratamiento sintomático del cáncer y como parte de los esquemas de antiemesis, que pueden enmascarar un hipocorticismo (24). Tanto es así, que en un estudio llevado a cabo por Redman et al. (25) con quince pacientes con infiltración suprarrenal bilateral, se demostró analíticamente hipofunción en el 33\% de los casos, por lo que algunos autores recomiendan iniciar tratamiento sustitutivo corticoideo tan pronto como se haga el diagnóstico de metástasis suprarrenales, independientemente de su tamaño $(11,26)$.

En el ámbito de la Oncología no sólo se han descrito casos de insuficiencia suprarrenal debidos a infiltración adrenal sino también secundarios a tratamientos farmacológicos con acetato de megestrol (27), suramina (28), interleucina-6 (29), inter- leucina-2 (30) y 5-fluorouracilo (31), a tratamiento radioterápico abdominal y a maniobras invasivas como la quimioembolización hepática (32).

En resumen, la enfermedad de Addison debe contemplarse dentro del diagnóstico diferencial de todo síndrome general en pacientes con cáncer, sobre todo si tienen afectación metastásica suprarrenal y existe un antecedente estresante. Una precoz confirmación de esta entidad, probablemente infradiagnosticada, puede prevenir su debut clínico como crisis addisoniana evitando así el mal pronóstico que conlleva. El tratamiento esteroideo sustitutivo con dosis bajas de hidrocortisona, asociando o no mineralocorticoides, es sencillo y consigue un buen control sintomático ofreciendo una mejoría sustancial de la calidad de vida. Queda aún por determinar la verdadera incidencia de insuficuencia suprarrenal por metástasis adrenales y la necesidad o no de iniciar tratamiento sustitutivo en el momento del diagnóstico de éstas, independientemente de la existencia de síntomas o de la confirmación bioquímica.

\section{Bibliografía}

1. Oelkers W. Adrenal insufficiency. N Eng J Med 1996; 335: 1206-12.

2. Werbel SS, Ober KP. Acute adrenal insufficiency. Endocrinology and Metabolism Clinics of North America 1993; 22: 303-27.

3. Williams GH, Dluhy RG. Enfermedades de la corteza suprarrenal. En: Isselbacher KI, Braunwald E, Wilson JD, Martin JB, Fauci AS, Kasper DL ed. Principios de Medicina Interna. $13^{\circ}$ ed. InteramericanaMcGrau-Hill, 1994: 2251-77.

4. Astorga Jiménez R, Silva Manzano H. Insuficiencia adrenocortical. Hipoaldosteronismo. Concepto, etiopatogenia y manifestaciones clínicas. Medicine 1997; 7 (46): 2005-10.

5. Gamelin E, Beldent V, Rousselet MC, Rieux D, Rohmer V, Ifrah N et al. Non-Hodgkin's Iymphoma presenting with primary adrenal insufficiency: a disease with an underestimated frequency? Cancer 1992; 69: 2333-36.

6. Kung AWC, Pun KK, Lam K, Wang C, Leung CY. Addisonian crisis as presenting feature in malignancies. Cancer 1990; 65: 177-79.

7. Gavilán Villarejo I, Ortego Rojo J, Escobar Jiménez L, Aguilar Diosdado M. Empleo de glucocorticoides en la insuficiencia suprarrenal. Manejo clínico de la insufucuencia suprarrenal iatrógena. Medicine 1997; 7 (60): 2761-70.

8. Efremidis SC, Harsoulis F, Douma S, Zafiriadou E, Zamboulis C, Kou ri A. Adrenal insufficiency with enlarged adrenals. Abdom Imaging 1996; 21: 168-71.

9. Sahún de la Vega M, Villabona Artero C, Montana Mías E, Fernández Real JM, Soler Ramón J. La importancia de la tomografía axial computerizada en el diagnóstico etiológico de la enfermedad de Addison. Rev Clin Esp 1992; 190: 177-80.

10. Mor F, Lahav M, Kipper E, Wysenbeek AJ. Addison's disease due to metastasis to the adrenal glands. Postgrad Med J 1985; 61: 637-9.

11. Levy NT, Young WF Jr, Habermann TM, Strickler JG, Carney JA, Stanson AW. Adrenal insufficiency as a manifestation of disseminated non-Hodgkin's Iymphoma. Mayo Clinic Proc 1997; 72 (9): 818-22.

12. Serrano S, Tejedor L, García B, Hallal H, Polo JA, Alguacil G. Addisonian crisis as presenting feature of bilateral primary adrenal Iymphoma. Cancer 1993; 71: 4040-3.

13. Feinmann C, Gillet R, Irving MH. Hodgkin's disease presenting with hypoadrenalism. Br Med J 1976; 2: 455-6.

14. Guzzini F, Cozzi C, Cortese F, Gasparini P, Neri V, Pace L. Adrenal failure due to bilateral metastases of relapsing lung carcinoma. Report of two cases. Tumori 1989; 75: 634-36.

15. Heuson JC. Addison's disease secondary to occult metastatic seminoma. Infraction of the adrenals as probable mechanism of their destruction. Cancer 1966; 19: 1754-59.

16. Kennedy RL, Ball RY, Dixon AK, Simon AT. Metastatic transitional cell carcinoma of the bladder causing Addison's disease. J Urol 1987; 137: $986-8$.
17. Lardinois CK, Zeng CL, Marshall MK. Acute adrenal insufficiency in association with pancreatic carcinoma. Arch Fam Med 1993; 2: 1194-7.

18. Omoigui NA, Cave WT Jr, Chang AY. Adrenal insufficiency. A rare initial sign of metastatic colon carcinoma. J Clin Gastroenterol 1987; 9: 470-4.

19. Hasan RI, Yonan NA, Lawson RA. Adrenal insufficiency due to bilateral metastases from oat-cell carcinoma of the oesophagus. Eur J Cardiothorac Surg 1991; 5: 336-7.

20. Cedermark BJ, Blumenson LE, Pickren JW, Elias EG. The significance of metastases to the adrenal gland from carcinoma of the stomach and esophagus. Surg Gynecol Obstet 1977; 145: 41-8.

21. Goffman TE, Schechter GP, McKeen EA, Mariani-Constantini R, Schein PS. Renal cell carcinoma causing a selective mineralocorticoid insufficiency. J Urol 1982; 128:370-1.

22. Chung HT, Wong KL, Liang RH, Loke SL, Liu HW, Tso WK et al. Non-Hodgkin's Iymphoma as a cause of hypoadrenalism. Aust N Z J Med 1987; 17: 605-7.

23. Sone H, Okuda Y, Nakamura Y, Asano M, Kawakami Y, Kawai K, Yamashita K. Primary adrenal Iymphoma presenting as addisonian crisis. Pitfalls in the diagnosis of bilateral adrenal swelling. Horm Metab Res 1996; 28: 116

24. Fuentes Jiménez FJ, Reyes Reyes F, Torres Serrano F, Blanco Negredo M, Jiménez Pérez JA. Crisis de Addison como manifestación inicial de tumor suprarrenal metastásico bilateral. An Med Intern 1995; 12: 619-20.

25. Redman BG, Pazdur R, Zingas AP, Loredo R. Prospective evaluation of adrenal insufficiency in patients with adrenal metastasis. Cancer 1987; 60: 103-7.

26. Seidenwurm DJ, Elmer EB, Kaplan LM, Williams EK, Morris DG, Hoffman AR. Metastases to the adrenal glands and the depelopment of Addison's disease. Cancer 1984; 54: 552-7.

27. Subramanian S, Goker H, Kanji A, Sweeney H. Clinical adrenal insuffi ciency in patients receiving megestrol therapy. Arch Intern Med 1997; 157: 1008-11.

28. Kobayashi K, Weiss RE, Vogelzang NJ, Vokes EE, Janisch L, Ratain MJ. Mineralocorticoid insufficiency due to suramin therapy. Cancer 1996; 78: 2411-20.

29. Chung U, Tanaka Y, Fujita T. Association of interleukin-6 and hypoaldosteronism in patients with cancer. N Engl J Med 1996; 334: 473.

30. Vandermolen LA, Smith JW 2nd, Longo DL, Steis RG, Kremers P, Sznol M. Adrenal insufficiency and interleukin-2 therapy. Ann Intern Med 1989 15; 111: 185.

31. Harris LN, Ragaz J. Ventricular arrhythmia and adrenal insufficiency after fluorouracil: new side effects of an old drug. J Natl Cancer Inst 1993; 85: 326-8.

32. Redd DC, Soulen MC, Crooks GW. Bilateral adrenal hemorrhage resulting in acute adrenal insuflciency as an unusual complication of hepatic arterial chemoembolization. J Vasc Interv Radiol 1998; 9: 271-4. 